\title{
Chirality groups of maps and hypermaps
}

\author{
Antonio Breda D'Azevedo • Gareth Jones • \\ Roman Nedela $\cdot$ Martin Škoviera
}

Received: 5 December 2005 / Accepted: 16 April 2008 / Published online: 1 May 2008 (C) Springer Science+Business Media, LLC 2008

\begin{abstract}
Although the phenomenon of chirality appears in many investigations of maps and hypermaps, no detailed study of chirality seems to have been carried out. Chirality of maps and hypermaps is not merely a binary invariant but can be quantified by two new invariants - the chirality group and the chirality index, the latter being the size of the chirality group. A detailed investigation of the chirality groups of orientably regular maps and hypermaps will be the main objective of this paper. The most extreme type of chirality arises when the chirality group coincides with the monodromy group. Such hypermaps are called totally chiral. Examples of these are constructed by considering appropriate "asymmetric" pairs of generators of certain non-abelian simple groups. We also show that every finite abelian group is the chirality group of some hypermap, whereas many non-abelian groups, including symmetric and dihedral groups, cannot arise as chirality groups.
\end{abstract}

A. Breda D’Azevedo supported in part by UI\&D Matemática e aplicações of University of Aveiro, through Program POCTI of FCT co-financed by the European Community fund FEDER.

R. Nedela supported in part by the Ministry for Education of the Slovak Republic, grant no. APVT 51027604.

M. Škoviera supported in part by APVT grant 51-027604 and by VEGA grant 1/3022/06.

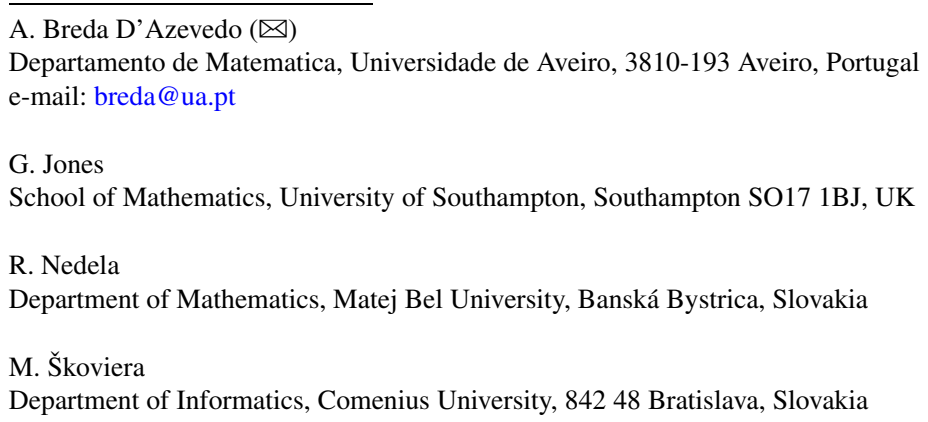


Keywords Map · Hypermap · Chiral · Asymmetric · Chirality group · Chirality index

Mathematics Subject Classification (2000) $05 \mathrm{C} 10 \cdot 05 \mathrm{C} 25 \cdot 20 \mathrm{~B} 25$

\section{Introduction}

The word chirality (meaning handedness) was introduced by William Thomson, better known as Lord Kelvin [22], as a property of a geometrical figure which occurs when "its image in a plane mirror, ideally realized, cannot be brought into coincidence with itself". There are many scientific examples of this phenomenon. For instance, chemists and biologists have discovered molecules which exist in two distinguishable enantiomers, which are mirror images of each other; in some cases, one form is beneficial whereas the other is poisonous. The phenomenon of chirality also appears in quantum mechanics, and more generally in theoretical physics.

These facts (see also $[1,5,15,19,24]$ ) provide a strong motivation for the study of chirality in mathematical models of physical structures. Many of these models are graphs embedded in the plane or 3-dimensional Euclidean space, or in more complex spaces endowed with a non-Euclidean geometry. For instance, fullerenes (recentlydiscovered positively-curved carbon structures) are modelled by 3-valent polyhedra with pentagonal and hexagonal faces, the pentagons being separated by hexagons; negatively-curved schwarzites arise if the pentagons are replaced with heptagons. These are special examples of maps.

Let us be more precise. A map on a surface is a cellular decomposition of a closed connected surface into 0 -cells called vertices, 1-cells called edges and 2-cells called faces. The vertices and edges of a map form its underlying graph, which is also connected. A map is said to be orientable if the supporting surface is orientable, and is oriented if one of two possible orientations of the surface has been specified. An automorphism of a map is an automorphism of the underlying graph which extends to a self-homeomorphism of the underlying surface. Map automorphisms split naturally into two classes, orientation-preserving and orientation-reversing automorphisms. We say that a map is chiral if it admits no orientation-reversing automorphism. These concepts extend naturally to hypermaps, generalisations of maps which we shall explain later.

Although the phenomenon of chirality appears in many investigations of maps and hypermaps (see Coxeter and Moser [12] for instance), no detailed study of chirality seems to have been carried out. A starting-point which led us to such a project was the observation that chirality of maps and hypermaps is not merely a binary invariant but can be quantified. Even more surprising was the fact that different approaches to measuring chirality lead to equivalent definitions, giving rise to related new invariants associated with any (hyper)map — the chirality group and the chirality index, the latter being the size of the chirality group. A detailed investigation of the chirality groups of maps and hypermaps will be the main objective of this paper.

In the investigation of maps and hypermaps it is often convenient to replace the topological objects with their combinatorial counterparts. Indeed, it is well-known 
that a map $\mathcal{H}$ on an orientable surface can be described by two permutations $R$ and $L$ acting on the set of darts (directed edges, or arcs). The permutation $R$ cyclically permutes the darts based at the same vertex, consistently with a chosen orientation of the surface, while $L$ interchanges the two oppositely directed darts sharing the same edge. Thus the orientation of the map can be encoded by the choice of $R$ (the other possibility being $R^{-1}$ ), and our maps are by definition oriented (or "polarized" using the language of physicists). By connectivity, the action of the group $\operatorname{Mon}(\mathcal{H})=$ $\langle R, L\rangle$ is transitive on the set of darts of $\mathcal{H}$. A hypermap is obtained if we simply relax the requirement $L^{2}=1$ by allowing $L$ to be of any order, so maps are particular examples of hypermaps. With the above notation $\mathcal{H}$ is said to be mirror symmetric or reflexible if the assignment $R \mapsto R^{-1}, L \mapsto L^{-1}$ extends to a group automorphism of $\langle R, L\rangle$; if it does not extend, we say that $\mathcal{H}$ is chiral.

Clearly, any hypermap $\mathcal{H}$ covers some reflexible hypermap $\mathcal{K}$, because in the worst case we can take $\mathcal{K}$ to be the trivial map consisting of one dart and a vertex attached to it. For simplicity, let us assume that the actions of $\operatorname{Mon}(\mathcal{H})$ and $\operatorname{Mon}(\mathcal{K})$ are both regular. Then the covering $\mathcal{H} \rightarrow \mathcal{K}$ arises from factoring out a certain normal subgroup of $\operatorname{Mon}(\mathcal{H})$. The minimal subgroup $X(\mathcal{H}) \unlhd \operatorname{Mon}(\mathcal{H})$ such that $\mathcal{H} / X(\mathcal{H})$ is a reflexible hypermap is called the chirality group of $\mathcal{H}$. It is straightforward that $\mathcal{H}$ is chiral if and only if $X(\mathcal{H})$ is nontrivial. There is a dual approach to the definition of the chirality group, obtained by considering the smallest reflexible hypermap which covers $\mathcal{H}$. It is proved in Section 3 that these two approaches are equivalent.

In general, $|X(\mathcal{H})| \leq|\operatorname{Mon}(\mathcal{H})|$. The most extreme type of chirality arises when $X(\mathcal{H})=\operatorname{Mon}(\mathcal{H})$; such hypermaps, called totally chiral, are studied in Section 5 where we construct examples of them by considering an appropriate "asymmetric" pair of generators for some non-abelian simple group. In Section 6 we show that every finite abelian group is the chirality group of some hypermap, whereas it is shown in Section 7 that many non-abelian groups, including symmetric and dihedral groups, cannot arise as chirality groups. The general problem of characterising chirality groups remains open.

\section{Hypermaps}

By an oriented hypermap we mean a triple $\mathcal{H}=(D, R, L)$ where $D$ is a set of darts and $R$ and $L$ are two permutations generating a permutation group $\operatorname{Mon}(\mathcal{H})=$ $\langle R, L\rangle$, called the monodromy group of $\mathcal{H}$, acting transitively on $D$. The permutations $R$ and $L$ will be called the canonical generators of Mon $(\mathcal{H})$. The orbits of $R, L$ and $R L$ on $D$ will be called the hypervertices, hyperedges, and hyperfaces, respectively. (We will write actions on the left, and hence we will compose permutations from right to left.)

In general, the set of darts of a hypermap may be infinite, however, our main interest lies in finite hypermaps, those where the set $D$ (equivalently, the group $\operatorname{Mon}(\mathcal{H})$ ) is finite. All hypermaps in this paper will be finite unless the immediate context implies otherwise.

If $m, n$ and $k$ are the orders of the permutations $R, L$ and $R L$, respectively, then the type of $\mathcal{H}$ is the triple $(m, n, k)$, and its Euler characteristic is the number $\chi(\mathcal{H})=$ $|D|\left(\frac{1}{m}+\frac{1}{n}+\frac{1}{k}-1\right)$. 
An oriented hypermap $\mathcal{H}$ is an oriented map if $L$ is an involution. Since the maps and hypermaps considered in this paper will all be oriented, the adjective oriented will often be omitted.

A map is an algebraic abstraction of a topological map, that is, a cellular decomposition of a closed connected surface. Hypermaps generalize the notion of maps in a natural way, but their geometric representations are somewhat less natural; they are described, for example, in [9-11, 30].

An automorphism of a hypermap $\mathcal{H}=(D, R, L)$ is a permutation $\psi$ of $D$ commuting with both $R$ and $L$. It is straightforward to see that the automorphism group Aut $(\mathcal{H})$ of $\mathcal{H}$ acts semi-regularly on the set of darts, so that $|\operatorname{Aut}(\mathcal{H})|$ divides $|D|$. If equality holds, the action is regular, and consequently $\mathcal{H}$ is called an orientably regular hypermap.

If $\mathcal{H}=(D, R, L)$ and $\mathcal{H}^{\prime}=\left(D^{\prime}, R^{\prime}, L^{\prime}\right)$ are hypermaps then a covering $\psi$ : $\mathcal{H} \rightarrow \mathcal{H}^{\prime}$ is a mapping $\psi: D \rightarrow D^{\prime}$ satisfying $\psi R=R^{\prime} \psi$ and $\psi L=L^{\prime} \psi$; note that a covering is necessarily surjective. We then say that $\mathcal{H}$ covers $\mathcal{H}^{\prime}$, and it follows that the assignment $R \mapsto R^{\prime}$ and $L \mapsto L^{\prime}$ extends to a canonical epimorphism $\operatorname{Mon}(\mathcal{H}) \rightarrow \operatorname{Mon}\left(\mathcal{H}^{\prime}\right)$ of the monodromy groups. If $\psi$ is an injective covering, we have an isomorphism $\mathcal{H} \cong \mathcal{H}^{\prime}$. A covering $\mathcal{H} \rightarrow \mathcal{H}^{\prime}$ is smooth if both hypermaps have the same type.

Let $\Delta$ denote the free product

$$
\Delta=\left\langle r_{0}, r_{1}, r_{2} \mid r_{0}^{2}=r_{1}^{2}=r_{2}^{2}=1\right\rangle
$$

and let $\Delta^{+}=\left\langle r_{1} r_{2}, r_{2} r_{0}\right\rangle$ be its "even word subgroup", the subgroup consisting of the words of even length in the generators. The canonical generators of $\Delta^{+}$will be denoted by $\rho=r_{1} r_{2}$ and $\lambda=r_{2} r_{0}$. Observe that the triple $\mathcal{U}=\left(\Delta^{+}, \rho, \lambda\right)$, with $\rho$ and $\lambda$ acting on $\Delta^{+}$by left translation, is a hypermap (clearly, an infinite one) which we will call the universal hypermap.

For any hypermap $\mathcal{H}=(D, R, L)$, finite or infinite, there is an epimorphism $\mu: \Delta^{+} \rightarrow \operatorname{Mon}(\mathcal{H})$ sending $\rho$ to $R$ and $\lambda$ to $L$. Consequently, $\mathcal{H}$ can be identified with the hypermap $\left(\Delta^{+} / H, \bar{\rho}, \bar{\lambda}\right)$ whose darts are the left cosets of the subgroup $H \leq \Delta^{+}, H$ (which may or may not be normal in $\Delta^{+}$) being the preimage of the stabiliser of a dart in $\mathcal{H}$ under the action of Mon $(\mathcal{H})$, and $\bar{\rho}(x H)=\rho x H$ and $\bar{\lambda}(x H)=\lambda x H$. With some abuse of notation, $\mu: \mathcal{U} \rightarrow\left(\Delta^{+} / H, \bar{\rho}, \bar{\lambda}\right) \cong \mathcal{H}$ is a hypermap covering. Thus the monodromy group of any oriented hypermap is a quotient of $\Delta^{+}$, and oriented hypermaps correspond to subgroups of $\Delta^{+}$. Any subgroup $H \leq \Delta^{+}$for which $\left(\Delta^{+} / H, \bar{\rho}, \bar{\lambda}\right) \cong \mathcal{H}$ will be called a hypermap subgroup for $\mathcal{H}$.

In the following statement we summarize some well-known facts on representations of hypermaps by hypermap subgroups (see [13]).

Lemma 1 Let $\mathcal{H}, \mathcal{H}_{1}$ and $\mathcal{H}_{2}$ be oriented hypermaps (not necessarily finite). Then the following statements hold:

(i) $\mathcal{H}_{1}$ covers $\mathcal{H}_{2}$ if and only if there are subgroups $H_{1} \leq H_{2} \leq \Delta^{+}$such that $\mathcal{H}_{1} \cong\left(\Delta^{+} / H_{1}, R_{1}, L_{1}\right)$ and $\mathcal{H}_{2} \cong\left(\Delta^{+} / H_{2}, R_{2}, L_{2}\right)$ where $R_{i}\left(x H_{i}\right)=\rho x H_{i}$ and $L_{i}\left(x H_{i}\right)=\lambda x H_{i}$ for $i=1,2$;

(ii) $\mathcal{H}_{1} \cong \mathcal{H}_{2}$ if and only if the corresponding hypermap subgroups are conjugate in $\Delta^{+}$ 
(iii) $\mathcal{H}$ is orientably regular if and only if there exists a normal subgroup $N \unlhd \Delta^{+}$ such that $\mathcal{H} \cong\left(\Delta^{+} / N, \rho N, \lambda N\right)$. Thus orientably regular hypermaps correspond to normal subgroups of $\Delta^{+}$.

While automorphisms of oriented hypermaps give rise to orientation-preserving self-homeomorphisms of the underlying surface, a hypermap may admit external symmetries coming from self-homeomorphisms which change the orientation of the surface. Such symmetries are called mirror symmetries (or reflections, or inversions). More precisely, a permutation $\psi$ of $D$ will be called a mirror symmetry of an oriented hypermap $\mathcal{H}=(D, R, L)$ if $\psi R=R^{-1} \psi$ and $\psi L=L^{-1} \psi$. An orientably regular hypermap admitting mirror symmetries is said to be reflexible or regular, since the automorphism group acts regularly on flags (triples formed by a mutually incident hypervertex, hyperedge and hyperface). On the other hand, an orientably regular hypermap with no mirror symmetries will be called a chiral hypermap. Observe that conjugation by $r_{2}$ induces an automorphism of $\Delta^{+}$inverting its generators $\rho$ and $\lambda$. Since a hypermap subgroup $H$ of an orientably regular hypermap $\mathcal{H}$ is normal in $\Delta^{+}$, its conjugates in $\Delta$ are $H$ and $H^{r_{0}}=H^{r_{1}}=H^{r_{2}}$. Let $H^{r}$ denote this common conjugate $H^{r_{i}}$. It is straightforward to see that $H^{r}$ is a hypermap subgroup of the mirror image $\mathcal{H}^{r}=\left(D, R^{-1}, L^{-1}\right)$, so $\mathcal{H}$ is reflexible if and only if $H^{r}=H$, or equivalently $H$ is a normal subgroup of $\Delta$.

\section{The chirality group and chirality index of a hypermap}

The aim of this section is to introduce invariants which in some sense measure the mirror asymmetry of a hypermap.

Let $\mathcal{H}$ be an orientably regular hypermap with hypermap subgroup $H$, a normal subgroup of $\Delta^{+}$. Then the largest normal subgroup of $\Delta$ contained in $H$ is the group $H_{\Delta}=H \cap H^{r}$, and the smallest normal subgroup of $\Delta$ containing $H$ is the group $H^{\Delta}=H H^{r}$. The corresponding hypermaps $\mathcal{H}_{\Delta}$ and $\mathcal{H}^{\Delta}$ are respectively the smallest reflexible hypermap that covers $\mathcal{H}$, and the largest reflexible hypermap that is covered by $\mathcal{H}$. In particular, if $\mathcal{H}$ is finite, so is $\mathcal{H}_{\Delta}$, since the intersection of two subgroups of finite index also has finite index.

Proposition 2 The four groups $H^{\Delta} / H, H / H_{\Delta}, H^{\Delta} / H^{r}$ and $H^{r} / H_{\Delta}$ are all isomorphic to each other.

Proof The third isomorphism theorem gives

$$
H^{\Delta} / H=H H^{r} / H \cong H^{r} /\left(H \cap H^{r}\right)=H^{r} / H_{\Delta},
$$

and similarly $H^{\Delta} / H^{r} \cong H / H_{\Delta}$. Conjugation by a generator $r_{i}$ of $\Delta$ induces isomorphisms $H^{\Delta} / H \cong H^{\Delta} / H^{r}$ and $H / H_{\Delta} \cong H^{r} / H_{\Delta}$.

We will call this common group the chirality group $X(\mathcal{H})$ of $\mathcal{H}$, and its order the chirality index $\kappa=\kappa(\mathcal{H})$ of $\mathcal{H}$. Thus $\mathcal{H}$ is reflexible if and only if $\kappa=1$, and in general $X(\mathcal{H})$ and $\kappa(\mathcal{H})$ can be regarded as algebraic and numerical measures of how far 
$\mathcal{H}$ deviates from being reflexible. We will restrict our attention to orientably regular hypermaps, since in the general case of an orientable hypermap one has to consider two conjugacy classes of subgroups $H$ and $H^{r}$ of $\Delta^{+}$, rather than two normal subgroups, so it is less clear how to define $\kappa(\mathcal{H})$ and $X(\mathcal{H})$.

Theorem 3 Let $\mathcal{H}$ be an orientably regular hypermap with chirality index $\kappa$. Then $\mathcal{H}_{\Delta} \rightarrow \mathcal{H}$ and $\mathcal{H} \rightarrow \mathcal{H}^{\Delta}$ are both $\kappa$-sheeted regular coverings with covering transformation group isomorphic to the chirality group $X(\mathcal{H})$. Moreover, the covering $\mathcal{H}_{\Delta} \rightarrow \mathcal{H}$ is smooth.

Proof Let $H$ be the hypermap subgroup for $\mathcal{H}$. Since $H_{\Delta}$ is normal in $H$ and $H$ is normal in $H^{\Delta}$, these coverings are both regular, being induced by the groups $H / H_{\Delta}$ and $H^{\Delta} / H$ of automorphisms of $\mathcal{H}_{\Delta}$ and $\mathcal{H}$. By Proposition 2 the corresponding covering transformation groups $H / H_{\Delta}$ and $H^{\Delta} / H$ are isomorphic, and they coincide with $X(\mathcal{H})$. The number of sheets of the covering is the index $\left|H: H_{\Delta}\right|$ or $\left|H^{\Delta}: H\right|$, equal in each case to $|X(\mathcal{H})|=\kappa$.

Since $r_{2}^{-1} \rho r_{2}=\rho^{-1}, r_{2}^{-1} \lambda r_{2}=\lambda^{-1}$, and $r_{2}^{-1}(\rho \lambda) r_{2}=\lambda(\rho \lambda)^{-1} \lambda^{-1}$, the same powers of $\rho, \lambda$ or $\rho \lambda$ lie in $H$ as in $H^{r}$, and hence as in $H \cap H^{r}=H_{\Delta}$. Thus $\mathcal{H}_{\Delta}$ has the same type as $\mathcal{H}$, and is therefore a smooth covering of $\mathcal{H}$, since in any covering of hypermaps, branching can occur only at the hypervertices, hyperedges or hyperfaces.

In general, the covering $\mathcal{H} \rightarrow \mathcal{H}^{\Delta}$ need not be smooth, since $H^{\Delta}$ may contain powers of $\rho, \lambda$ or $\rho \lambda$ which do not lie in $H$ or $H^{r}$; indeed, we shall see in Section 5 that there are nontrivial hypermaps $\mathcal{H}$ for which $\mathcal{H}^{\Delta}$ is the trivial hypermap, of type $(1,1,1)$.

Corollary 4 If $\mathcal{H}$ is an orientably regular hypermap with chirality index $\kappa$, then $\chi\left(\mathcal{H}_{\Delta}\right)=\kappa \chi(\mathcal{H})$.

Proposition 5 The chirality group $X(\mathcal{H})$ of each orientably regular hypermap $\mathcal{H}$ is isomorphic to a normal subgroup of the monodromy group Mon $(\mathcal{H})$.

Proof $X(\mathcal{H}) \cong H^{\Delta} / H \leq \Delta^{+} / H \cong \operatorname{Mon}(\mathcal{H})$. By Theorem 3, $X(\mathcal{H})$ is normal in $\operatorname{Mon}(\mathcal{H})$.

Since the number of darts in an orientably regular hypermap coincides with the order of the monodromy group, Proposition 5 and Lagrange's Theorem now imply:

Corollary 6 The chirality index of any orientably regular hypermap divides the number of darts.

The following example shows that the chirality index of a hypermap can be arbitrarily large. 
Example 1 (see [4]) Consider the metacyclic group

$$
G=\left\langle a, b \mid a^{n}=1, b^{m}=a^{s}, b a b^{-1}=a^{r}\right\rangle
$$

of order $m n$, where $r s \equiv s(\bmod n)$ and $r^{m} \equiv 1(\bmod n)$ for some $m>2$. If we regard $a$ and $b$ as the canonical generators for an orientably regular hypermap $\mathcal{H}=(G, a, b)$ with monodromy group $G$, then it follows from the definition of the chirality group $X(\mathcal{H})$ as $H^{\Delta} / H$ that $X(\mathcal{H})$ can be interpreted as the smallest normal subgroup $N$ of $G$ such that the assignment $a \mapsto a^{-1}$ and $b \mapsto b^{-1}$ induces an automorphism of $G / N$. We obtain this quotient from $G$ by adding the extra relations formed from those of $G$ by replacing $a$ and $b$ with their inverses. In this case, it is sufficient to add $b^{-1} a^{-1} b=a^{-r}$, or equivalently, $b^{-1} a b=a^{r}$, so that $a=b\left(b^{-1} a b\right) b^{-1}=\left(a^{r}\right)^{r}=a^{r^{2}}$ in $G / N$. Thus $a^{r^{2}-1}=1$ in $G / N$, so in $G$ it follows that $K=\left\langle a^{r^{2}-1}\right\rangle$ is a subgroup of $N$. On the other hand, it is easy to see that $K$ is a normal subgroup of $G$ such that $G / K$ is invariant under replacement of the generators with their inverses. By minimality $N=K$, so $X(\mathcal{H}) \cong\left\langle a^{r^{2}-1}\right\rangle$ and $\kappa(\mathcal{H})=n / \operatorname{gcd}\left(n, r^{2}-1\right)$; since $m>2$, this can be arbitrarily large. For instance, given any $m>2$ we could choose a prime $n \equiv 1(\bmod m)$ (there are infinitely many, by Dirichlet's Theorem on primes in arithmetic progression), so that there is an integer $r$ of multiplicative order $m \bmod n$; if we put $s=0$ then $G$ is a semidirect product of $\langle a\rangle \cong C_{n}$ by $\langle b\rangle \cong C_{m}$, and $\kappa(\mathcal{H})=n$ since $\operatorname{gcd}\left(n, r^{2}-1\right)=1$.

\section{Normal subgroups of $\Delta$ and $\Delta^{+}$}

In order to study chirality groups further we need some elementary results telling us which normal subgroups of $\Delta^{+}$are also normal in $\Delta$.

The following result is obvious.

Lemma 7 Let $N$ be a normal subgroup of $\Delta^{+}$, and let $G=\Delta^{+} / N$. Then the following are equivalent:

(i) $N$ is normal in $\Delta$;

(ii) $N^{r}=N$;

(iii) $G$ has an automorphism inverting both of its canonical generators;

(iv) the hypermap corresponding to $N$ is reflexible.

Let us call a generating pair $x, y$ for a group $G$ symmetric if there is an automorphism of $G$ inverting both $x$ and $y$, and asymmetric otherwise. Let us call a 2 -generator group $G$ strongly symmetric if all its generating pairs are symmetric. By Lemma 7, this condition means that every normal subgroup of $\Delta^{+}$with quotient group $G$ is normal in $\Delta$, or equivalently, every orientably regular hypermap with monodromy group $G$ is reflexible.

Proposition 8 The following groups are all strongly symmetric: 2-generator abelian groups, dihedral groups, $P S L_{2}(q)$ for any prime power $q$, and the symmetric groups $S_{2}, S_{3}, S_{4}$ and $S_{5}$. 
Proof Every abelian group has an automorphism inverting all its elements. If two elements generate a dihedral group, they are either two reflections or a reflection and a rotation; in the first case they are both inverted by the identity automorphism, and in the second case they are inverted by conjugation by the reflection. It follows easily from [23, Theorem 3] that if $x, y$ and $x^{\prime}, y^{\prime}$ are two generating pairs for $\operatorname{PSL}_{2}(q)$ with $\operatorname{tr} x=\operatorname{tr} x^{\prime}, \operatorname{tr} y=\operatorname{tr} y^{\prime}$ and $\operatorname{tr} x y=\operatorname{tr} x^{\prime} y^{\prime}$, then there is an automorphism sending $x$ to $x^{\prime}$ and $y$ to $y^{\prime}$; now $\operatorname{tr} x=\operatorname{tr} x^{-1}, \operatorname{tr} y=\operatorname{tr} y^{-1}$ and $\operatorname{tr} x y=\operatorname{tr} y x=\operatorname{tr}(y x)^{-1}=$ $\operatorname{tr} x^{-1} y^{-1}$, so $x, y$ form a symmetric pair (see [27] for this and related arguments). The small symmetric groups are dealt with by a routine inspection of their generating pairs.

Note that this shows that the alternating groups $A_{n}$ are strongly symmetric for $n \leq 6$ since $A_{1}$ and $A_{2}$ are trivial, $A_{3} \cong C_{3}, A_{4} \cong P S L_{2}(3), A_{5} \cong P S L_{2}(4)$ and $A_{6} \cong P S L_{2}$ (9). If $n \geq 6$ then $S_{n}$ is not strongly symmetric, since $x=(12)$ (345) and $y=(12 \ldots n)$ form an asymmetric pair of generators; see also Theorem 13 for $A_{n}, n \geq 7$.

An immediate consequence of Proposition 8 and Lemma 7 is the following:

Corollary 9 If $N$ is a normal subgroup of $\Delta^{+}$such that $\Delta^{+} / N$ is abelian or dihedral or isomorphic to $\mathrm{PSL}_{2}(q)$ or $S_{n}$ for some $n \leq 5$, then $N$ is normal in $\Delta$.

Example 2 As an application of Corollary 9, the following simple example shows that every elementary abelian group of prime power order $q=p^{e}>4$ can arise as a chirality group. (We will extend this result later to all finite abelian groups, but for this we need a more complicated construction.) Let $G$ be the 1-dimensional affine group $A G L_{1}(q)$ over the field $F_{q}$ of order $q$, consisting of the transformations $t \mapsto$ $a t+b$ where $a, b \in F_{q}$ and $a \neq 0$. This is a split extension of the translation group $B=\left\{t \mapsto t+b \mid b \in F_{q}\right\}$, an elementary abelian group of order $q$ isomorphic to the additive group of $F_{q}$, by the group $A=\left\{t \mapsto a t \mid a \in F_{q}^{*}\right\}$, isomorphic to the multiplicative group $F_{q}^{*}=F_{q} \backslash\{0\}$ of $F_{q}$. Let $x$ and $y$ be the elements $t \mapsto c t$ and $t \mapsto t+1$ of $G$, where $c$ generates the (cyclic) group $F_{q}^{*}$. Then $x$ and $y$ generate $G$, so the epimorphism $\theta: \Delta^{+} \rightarrow G, R \mapsto x, L \mapsto y$ realises $G$ as the monodromy group of an orientably regular hypermap $\mathcal{H}$, with hypermap subgroup $H=\operatorname{ker} \theta \leq \Delta^{+}$.

If $\Gamma$ denotes the Galois group of $F_{q}$ (over its prime field $F_{p}$ ), then Aut $G$ can be identified with $A \Gamma L_{1}(q)$, the group of transformations $t \mapsto a t^{\gamma}+b$ where $a \neq 0$ and $\gamma \in \Gamma$; this contains $G$ as a normal subgroup, and induces automorphisms of $G$ by acting by conjugation. It follows that $x$ is inverted by an automorphism of $G$ if and only if $c$ is equivalent to $c^{-1}$ under $\Gamma$. Now $\Gamma$ is a cyclic group of order $e$, generated by the Frobenius automorphism $t \mapsto t^{p}$ of $F_{q}$, so the images of $c$ under $\Gamma$ are the powers $c^{p^{i}}$ for $i=1, \ldots, e$. Thus $c$ is equivalent to $c^{-1}$ if and only if $c^{p^{i}+1}=1$ for some $i$, or equivalently ( $\operatorname{since} c$ has order $p^{e}-1$ ) if $p^{e}-1$ divides $p^{i}+1$, which is impossible if $p^{e}>4$. Thus $\mathcal{H}$ is chiral for all $q>4$, so $H^{\Delta}>H$.

If $N$ denotes $\theta^{-1}(B)$ then $\Delta^{+} / N \cong G / B \cong A$, which is abelian, so $N$ is normal in $\Delta$ by Corollary 9 , and hence $H^{\Delta} \leq N$. Now $B$ is a minimal normal subgroup of $G$, so $N / H$ is a minimal normal subgroup of $\Delta^{+} / H$. Since $N \geq H^{\Delta}>H$ it follows that $H^{\Delta}=N$, so $\mathcal{H}$ has chirality group $X(\mathcal{H}) \cong N / H \cong B$. 


\section{Totally chiral hypermaps}

The most extreme type of chirality, and often the easiest to study, occurs when an orientably regular hypermap $\mathcal{H}$ is totally chiral, meaning that the chirality group of $\mathcal{H}$ coincides with its monodromy group. If $H$ is the map subgroup of $\Delta^{+}$corresponding to $\mathcal{H}$, then $\mathcal{H}$ is totally chiral if and only if $H^{\Delta}=\Delta^{+}$, so a totally chiral hypermap is one which covers no nontrivial reflexible hypermaps.

In this section we will study some examples of this phenomenon, before considering more general forms of chirality in the next section.

The next result implies that totally chiral hypermaps are rather rare, though we will give some infinite families of examples in Theorems 12, 13 and 14. A perfect group is one with no nontrivial abelian epimorphic images.

Corollary 10 The monodromy group of a totally chiral hypermap cannot have any nontrivial strongly symmetric group as an epimorphic image; in particular, it must be perfect.

Proof Let $\mathcal{H}$ be a totally chiral hypermap with monodromy group $\operatorname{Mon}(\mathcal{H})=\Delta^{+} / H$ for some $H \unlhd \Delta^{+}$. Assume, for a contradiction, that $\operatorname{Mon}(\mathcal{H})$ has a nontrivial strongly symmetric epimorphic image $G$. Then there exists a subgroup $N$ of $\Delta^{+}$ such that $H \leq N<\Delta$ and $G=\Delta^{+} / N$. Since $\Delta^{+} / N$ is strongly symmetric, we have $N \unlhd \Delta$ and hence $N N^{r}=N$. Therefore

$$
\Delta^{+}=H H^{r} \leq N N^{r}=N<\Delta^{+}
$$

which is a contradiction. In particular, since abelian groups are strongly symmetric, they cannot occur as nontrivial epimorphic images.

The most obvious examples of perfect groups are the nonabelian simple groups, so it makes sense to inspect these for examples of totally chiral hypermaps.

Lemma 11 If $X$ is a simple group with an asymmetric generating pair, then $X$ is the monodromy group of a totally chiral hypermap.

Proof Let $x$ and $y$ be an asymmetric generating pair for $X$, and let $H$ be the kernel of the epimorphism $\Delta^{+} \rightarrow X$ given by $\rho \mapsto x, \lambda \mapsto y$, so $X \cong \Delta^{+} / H$ is the monodromy group of the orientably regular hypermap $\mathcal{H}$ corresponding to $H$. By Lemma 7 we have $H^{r} \neq H$, so $H^{\Delta}$ is a normal subgroup of $\Delta^{+}$properly containing $H$. The simplicity of $X$ gives $H^{\Delta}=\Delta^{+}$, so $\mathcal{H}$ is totally chiral.

Some simple groups $X$ have an element $x$ which is not inverted by any automorphism; if there is a second element $y$ such that $x$ and $y$ generate $X$, then Lemma 11 implies that $X$ is the monodromy group of a totally chiral hypermap. Here we give two families of groups which illustrate this principle.

The Ree groups $\operatorname{Re}\left(3^{f}\right)={ }^{2} G_{2}\left(3^{f}\right)$ and the Suzuki groups $S z\left(2^{f}\right)={ }^{2} B_{2}\left(2^{f}\right)$ are defined for all odd $f \geq 1$, and are simple for $f>1$. They were first described in [25, $26]$ and [29] respectively, and a good account of their properties can also be found in Chapter XI of [18]. 
Theorem 12 For each odd $f>1$ there are totally chiral hypermaps with monodromy groups $\operatorname{Re}\left(3^{f}\right)$ and $S z\left(2^{f}\right)$.

Proof The Ree group $X=\operatorname{Re}\left(3^{f}\right)$, for odd $f>1$, is a simple epimorphic image of the triangle group $\Delta(2,3,7)$, and hence of $\Delta^{+}$, with generators $x$ and $y$ of orders 2 and 3. As shown in [26], $y$ is not inverted by any automorphism of $X$, so Lemma 11 applies. Similarly $S z\left(2^{f}\right)$ is a simple epimorphic image of $\Delta(2,4,5)$ for odd $f>1$, and the generator of order 4 is not inverted by any automorphism [29]. (See [20, Section 6] and [21, Section 6] for further details and for descriptions of the chiral hypermaps associated with these groups.)

Other simple examples of this phenomenon include the Mathieu groups $M_{p}$ for $p=11$ and 23: in each case an element of order $p$ is a member of a generating pair, but is not inverted by any automorphism (see [8] for details).

A more common phenomenon among simple groups is that both members $x$ and $y$ of a generating pair may be inverted by automorphisms, but no single automorphism inverts them both. To illustrate this we consider the alternating groups $A_{n}$, which are simple for all $n \geq 5$; here every element, having the same cycle structure as its inverse, is inverted by conjugation in $S_{n}$.

Theorem 13 For each $n \geq 7$ there is a totally chiral hypermap with monodromy group $A_{n}$.

Proof If $n$ is odd, let $X$ be the subgroup of the symmetric group $S_{n}$ generated by the permutations $x=(12 \ldots n)$ and $y=(124)$. These are both even, so $X \leq A_{n}$. Any $x$-invariant relation on $\{1,2, \ldots, n\}$ must be congruence mod $m$ for some $m$ dividing $n$, and $y$ preserves this only for $m=1$ and $n$, so $X$ is primitive. A primitive group containing a 3-cycle must contain $A_{n}$ [31, Theorem 13.3], so $X=A_{n}$. For $n \geq 7$ one can identify Aut $A_{n}$ with $S_{n}$, acting by conjugation on its normal subgroup $A_{n}$; the only permutations inverting $x$ are the reflections in the obvious dihedral group $D_{n}$ containing $x$, and these do not invert $y$, so Lemma 11 gives the required result.

If $n$ is even, one can take $x=(12 \ldots n-1)$ and $y=(1 n)(23)$ in $A_{n}$. The group $X=\langle x, y\rangle$ is clearly transitive; since $x$ fixes $n$ and has a single cycle on the remaining points, $X$ is doubly transitive and hence primitive. Now $X$ contains a 5-cycle $[x, y]=$ $x^{-1} y^{-1} x y=(1 n 342)$, and a theorem of Jordan [31, Theorem 13.9] states that a primitive group of degree $n$, containing a $p$-cycle for some prime $p \leq n-3$, must contain $A_{n}$. It follows that $X=A_{n}$ provided $n \geq 8$. Again there is no permutation in $S_{n}$ which inverts $x$ and $y$, so Lemma 11 completes the proof.

By the comment after Proposition 8, this result does not extend to $A_{n}$ for $n \leq 6$.

For a similar class of examples, we use the simple groups $P S L_{d}(q)$ and their covering groups $S L_{d}(q)$. Note that the case $d=2$ is excluded by Proposition 8 and Corollary 10, and similarly $P S L_{3}(2)=S L_{3}(2)$ is excluded since it is isomorphic to $\mathrm{PSL}_{2}(7)$.

Each $a \in G L_{d}(q)$ induces an inner automorphism $\iota_{a}: g \mapsto g^{a}=a^{-1} g a$ of $G L_{d}(q)$, each $\gamma \in \Gamma=\mathrm{Gal} F_{q}$ induces an automorphism by acting on matrix entries, and the adjoint mapping $g \mapsto g^{*}=\left(g^{T}\right)^{-1}$ is also an automorphism of $G L_{d}(q)$ 
(the graph automorphism in Lie algebra terminology). Every automorphism of $G L_{d}(q)$ is a composition of these, having the form $\alpha_{a, \gamma}: g \mapsto\left(g^{a}\right)^{\gamma}$ or $\beta_{a, \gamma}: g \mapsto$ $\left(\left(g^{*}\right)^{a}\right)^{\gamma}$. These automorphisms all induce automorphisms of $P G L_{d}(q), S L_{d}(q)$ and $P S L_{d}(q)$, and conversely all automorphisms of these groups arise in this way [14, Ch. IV]. Since every matrix is similar (that is, conjugate) to its transpose, every element of these groups is inverted by some automorphism. Nevertheless, it is usually possible to find an asymmetric generating pair in these groups.

Theorem 14 If $d \geq 3$ and if the prime power $q$ is sufficiently large (in terms of $d$ ) then there are totally chiral hypermaps with monodromy groups $S L_{d}(q)$ and $P S L_{d}(q)$.

Proof Let $x$ be a diagonal matrix in $X=S L_{d}(q)$, with spectrum $\Lambda \subset F_{q}$ consisting of $d$ distinct eigenvalues, so the centraliser of $x$ in $G L_{d}(q)$ consists of the diagonal matrices. We will show that if $q$ is sufficiently large then one can choose $\Lambda$ so that $x$ is a member of an asymmetric generating pair $x, y$ for $X$, with a similar result for their images $\bar{x}, \bar{y}$ in $\bar{X}=P S L_{d}(q)$.

The automorphisms $\iota_{a}$ preserve eigenvalues, the field-automorphisms act naturally on them, and the adjoint automorphism inverts them. The image $\bar{x}$ of $x$ in $\bar{X}$ is represented by the matrices $\omega x$ where $\omega^{d}=1$, and these have spectrum $\omega \Lambda$. For any fixed $d \geq 3$, if $q$ is sufficiently large one can choose $\Lambda$ to be distinct from the sets $\omega\left(\Lambda^{-1}\right)^{\gamma}$ where $\omega^{d}=1$ and $\gamma \in \Gamma$, and also from the sets $\omega \Lambda^{\gamma}$ except where $\omega=1$ and $\gamma=1$. (For example, one can take $\Lambda=\left\{\zeta, \zeta^{2}, \ldots, \zeta^{d-1}, \zeta^{-d(d-1) / 2}\right\}$ for sufficiently large $q$, where $\zeta$ generates $F_{q}^{*}$, noting that inversion and multiplication by $\omega$ preserve the multiset of ratios $\lambda / \mu$ for $\lambda, \mu \in \Lambda$; no such choice is possible for $d=2$ since the condition $\operatorname{det} x=1$ forces $\Lambda=\Lambda^{-1}$.) Our conditions on $\Lambda$ then ensure that the only automorphisms inverting $\bar{x}$ (and hence the only automorphisms inverting $x$ ) are the automorphisms $\beta_{a, 1}: g \mapsto\left(g^{*}\right)^{a}$ where $a$ commutes with $x$ and is therefore a diagonal matrix. A simple calculation shows that the proportion of elements $y \in X$ (or $\bar{y} \in \bar{X}$ ) inverted by any such $\beta_{a, 1}$ approaches 0 as $q \rightarrow \infty$, whereas recent results on random generation of simple groups [17] imply that the proportion of $\bar{y}$ such that $\langle\bar{x}, \bar{y}\rangle=\bar{X}$ approaches 1 . This means that for all sufficiently large $q, \bar{X}$ has an asymmetric generating pair $\bar{x}, \bar{y}$, so Lemma 11 shows that $\bar{X}$ is the monodromy group of a totally chiral hypermap, corresponding to the kernel $H$ of the obvious epimorphism $\Delta^{+} \rightarrow \bar{X}$.

Clearly $x, y$ form an asymmetric pair, since any automorphism of $X$ inverting them would induce an automorphism of $\bar{X}$ inverting $\bar{x}$ and $\bar{y}$; we now show that they generate $X$. The subgroup $S=\langle x, y\rangle$ maps onto $\bar{X}$, so $S Z=X$ where $Z=\operatorname{ker}(X \rightarrow$ $\bar{X})$ is the centre of $X$, and hence $S$ is normal in $X$ (since it commutes with $Z$ ); now $X / S=S Z / S \cong Z /(S \cap Z)$, which is abelian, and $X$ is perfect, so $S=X$ as required. This gives an epimorphism $\Delta^{+} \rightarrow X$ with kernel $K$ contained in $H$. Now $K^{\Delta}$ is a normal subgroup of $\Delta^{+}$, properly containing $K$, so because every proper normal subgroup of $X=S L_{d}(q)$ is contained in $Z$ we have $K^{\Delta} \leq H$ or $K^{\Delta}=\Delta^{+}$. The first case implies that $H / K^{\Delta}$ is the centre of $\Delta^{+} / K^{\Delta}$, so $H$ is normal in $\Delta$, which is false. Hence $K^{\Delta}=\Delta^{+}$, so the hypermap corresponding to $K$ is totally chiral, with monodromy group $X$. 
No doubt, a more careful examination of the families of finite simple groups would reveal further examples of totally chiral hypermaps.

Remark. The chiral maps up to genus 15 have abelian chirality groups (see [2]) and therefore they cannot be totally chiral. Among these is the Edmonds map (that is, the map C7.2 in [7]), a chiral map of genus 7 and size 56, for which the chirality group is elementary abelian of order 8 ; this is our Example 2 with $q=8$. Totally chiral hypermaps do not appear for small genera: the smallest genus given by our proof of Theorem 13 is 481 (with $n=7$ ) and by examining the computer classification of chiral maps and hypermaps of small genus recently released by Conder [6] we find that none of the chiral maps and hypermaps up to genus 101 is totally chiral.

\section{Chirality groups and direct products}

In this section we define a form of direct product of hypermaps, and we investigate how the chirality group of the product can be expressed in terms of the chirality groups of the factors. As an application of our general results we show that every finite abelian group is the chirality group of some hypermap.

Let $\mathcal{H}$ and $\mathcal{K}$ be orientably regular hypermaps with hypermap subgroups $H, K \leq$ $\Delta^{+}$. We define the least common cover $\mathcal{H} \vee \mathcal{K}$ and the greatest common quotient $\mathcal{H} \wedge \mathcal{K}$ of $\mathcal{H}$ and $\mathcal{K}$ to be the orientably regular hypermaps with hypermap subgroups $H \cap K$ and $\langle H, K\rangle=H K$ respectively. For example, we have $\mathcal{H}_{\Delta}=\mathcal{H} \vee \mathcal{H}^{r}$ and $\mathcal{H}^{\Delta}=\mathcal{H} \wedge \mathcal{H}^{r}$. Clearly, every common cover of $\mathcal{H}$ and $\mathcal{K}$ also covers $\mathcal{H} \vee \mathcal{K}$, and any common quotient of them is also a quotient of $\mathcal{H} \wedge \mathcal{K}$. The coverings $\mathcal{H} \vee \mathcal{K} \rightarrow$ $\mathcal{H}$ and $\mathcal{K} \rightarrow \mathcal{H} \wedge \mathcal{K}$ are regular with covering transformation group $H /(H \cap K) \cong$ $H K / K$, and the coverings $\mathcal{H} \vee \mathcal{K} \rightarrow \mathcal{K}$ and $\mathcal{H} \rightarrow \mathcal{H} \wedge \mathcal{K}$ are regular with covering transformation group $K /(H \cap K) \cong H K / H$.

We can write $\mathcal{H}=\left(D_{1}, R_{1}, L_{1}\right)$ and $\mathcal{K}=\left(D_{2}, R_{2}, L_{2}\right)$ for sets $D_{i}$ of darts, so that the actions $\rho \mapsto R_{i}, \lambda \mapsto L_{i}$ of $\Delta^{+}$on $D_{1}$ and $D_{2}$ induce a product action $\rho \mapsto R, \lambda \mapsto L$ of $\Delta^{+}$on the set $D=D_{1} \times D_{2}$, given by $R(x, y)=\left(R_{1} x, R_{2} y\right)$ and $L(x, y)=\left(L_{1} x, L_{2} y\right)$. If this action is transitive then it determines an orientably regular hypermap $\mathcal{H} \times \mathcal{K}=(D, R, L)$ called the oriented direct product of $\mathcal{H}$ and $\mathcal{K}$, with hypermap subgroup $H \cap K$. The following straightforward lemma tells us when this construction is possible (see [3]):

Lemma 15 If $\mathcal{H}$ and $\mathcal{K}$ are orientably regular hypermaps, then the following three conditions are equivalent:

(a) $\Delta^{+}$acts transitively on $D$;

(b) $\mathcal{H} \wedge \mathcal{K}$ is the trivial orientable hypermap, with one dart;

(c) $H K=\Delta^{+}$.

When these conditions are satisfied, we say that $\mathcal{H}$ and $\mathcal{K}$ are orientably orthogonal, and write $\mathcal{H} \perp \mathcal{K}$; then $\mathcal{H} \times \mathcal{K}$ exists, and is isomorphic to $\mathcal{H} \vee \mathcal{K}$, with monodromy group $\operatorname{Mon}(\mathcal{H} \times \mathcal{K})=\operatorname{Mon}(\mathcal{H}) \times \operatorname{Mon}(\mathcal{K})$. For example, $\mathcal{H}$ is totally chiral if and only if $\mathcal{H} \perp \mathcal{H}^{r}$, in which case $\mathcal{H}_{\Delta}=\mathcal{H} \times \mathcal{H}^{r}$ with $\operatorname{Mon}\left(\mathcal{H}_{\Delta}\right)=$ $\operatorname{Mon}(\mathcal{H}) \times \operatorname{Mon}\left(\mathcal{H}^{r}\right) \cong(\operatorname{Mon}(\mathcal{H}))^{2}$. 
Proposition 16 Let $\mathcal{H}$ and $\mathcal{K}$ be orientably regular hypermaps, with hypermap subgroups $H$ and $K$, such that $\mathcal{K}$ is totally chiral and covers $\mathcal{H}$. Then the product $\mathcal{L}=\mathcal{K} \times \mathcal{H}^{r}$ is an orientably regular hypermap with chirality group $X(\mathcal{L}) \cong H / K$.

Proof Since $\mathcal{K}$ is totally chiral and $K \leq H$, we have $K H^{r} \geq K K^{r}=\Delta^{+}$, so $K H^{r}=$ $\Delta^{+}$and hence $\mathcal{K} \perp \mathcal{H}^{r}$. Consequently, the product $\mathcal{L}=\mathcal{K} \times \mathcal{H}^{r}$ is a well-defined orientably regular hypermap with hypermap subgroup $L=K \cap H^{r}$. Using $L_{\Delta}=$ $K \cap L^{r}$, the third isomorphism theorem, and $K L^{r}=H$, we have $X(\mathcal{L}) \cong L^{r} / L_{\Delta}=$ $L^{r} /\left(K \cap L^{r}\right) \cong K L^{r} / K=H / K$.

We define two groups $A$ and $B$ to be mutually orthogonal, and write $A \perp B$, if they have no non-trivial common epimorphic images.

Lemma 17 Let $\mathcal{H}$ and $\mathcal{K}$ be orientably regular hypermaps.

(a) If $\operatorname{Mon}(\mathcal{H}) \perp \operatorname{Mon}(\mathcal{K})$, then $\mathcal{H} \perp \mathcal{K}$.

(b) If, in addition, $\mathcal{H}$ and $\mathcal{K}$ are totally chiral, so is $\mathcal{H} \vee \mathcal{K}$.

Proof (a) Let $H$ and $K$ be the hypermap subgroups of $\Delta^{+}$corresponding to $\mathcal{H}$ and $\mathcal{K}$. If $H K \neq \Delta^{+}$, then $\Delta^{+} / H K$ is a non-trivial epimorphic image of $\Delta^{+} / H \cong \operatorname{Mon}(\mathcal{H})$ and of $\Delta^{+} / K \cong \operatorname{Mon}(\mathcal{K})$, against our assumption.

(b) Since $\mathcal{H} \perp \mathcal{H}^{r}$, we have $\mathcal{H}_{\Delta}=\mathcal{H} \times \mathcal{H}^{r}$, and similarly for $\mathcal{K}_{\Delta}$. The groups $\operatorname{Mon}\left(\mathcal{H}_{\Delta}\right)=\operatorname{Mon}(\mathcal{H}) \times \operatorname{Mon}\left(\mathcal{H}^{r}\right) \cong(\operatorname{Mon}(\mathcal{H}))^{2}$ and $\operatorname{Mon}\left(\mathcal{K}_{\Delta}\right)=\operatorname{Mon}(\mathcal{K}) \times$ $\operatorname{Mon}\left(\mathcal{K}^{r}\right) \cong(\operatorname{Mon}(\mathcal{K}))^{2}$ are mutually orthogonal (since $\operatorname{Mon}(\mathcal{H})$ and $\operatorname{Mon}(\mathcal{K})$ are), so $\mathcal{H}_{\Delta} \perp \mathcal{K}_{\Delta}$ by (a). Now $(H \cap K)_{\Delta}=H_{\Delta} \cap K_{\Delta}$, so

$$
\begin{aligned}
\operatorname{Mon}(\mathcal{H} \vee \mathcal{K})_{\Delta} & =\operatorname{Mon}\left(\mathcal{H}_{\Delta}\right) \times \operatorname{Mon}\left(\mathcal{K}_{\Delta}\right) \\
& =\left(\operatorname{Mon}(\mathcal{H}) \times \operatorname{Mon}\left(\mathcal{H}^{r}\right)\right) \times\left(\operatorname{Mon}(\mathcal{K}) \times \operatorname{Mon}\left(\mathcal{K}^{r}\right)\right) \\
& =(\operatorname{Mon}(\mathcal{H}) \times \operatorname{Mon}(\mathcal{K})) \times\left(\operatorname{Mon}\left(\mathcal{H}^{r}\right) \times \operatorname{Mon}\left(\mathcal{K}^{r}\right)\right) \\
& =\operatorname{Mon}(\mathcal{H} \vee \mathcal{K}) \times \operatorname{Mon}\left((\mathcal{H} \vee \mathcal{K})^{r}\right)
\end{aligned}
$$

as required.

The converse of (a) is false: for instance, for each prime $p$ there are $p+1$ mutually orthogonal orientably regular hypermaps $\mathcal{H}$ with $\operatorname{Mon}(\mathcal{H}) \cong C_{p}$.

Corollary 18 Let $\mathcal{H}_{1}, \ldots, \mathcal{H}_{k}$ be orientably regular hypermaps.

(a) If the groups Mon $\left(\mathcal{H}_{i}\right)$ are pairwise orthogonal, then

$$
\mathcal{H}_{1} \vee \cdots \vee \mathcal{H}_{k}=\mathcal{H}_{1} \times \cdots \times \mathcal{H}_{k}
$$

(b) If, in addition, each hypermap $\mathcal{H}_{i}$ is totally chiral, then so is $\mathcal{H}_{1} \vee \cdots \vee \mathcal{H}_{k}$.

Proof This follows by induction on $k$ : since Mon $\left(\mathcal{H}_{i}\right) \perp$ Mon $\left(\mathcal{H}_{k}\right)$ for all $i<k$ we have $\operatorname{Mon}\left(\mathcal{H}_{1}\right) \times \cdots \times \operatorname{Mon}\left(\mathcal{H}_{k-1}\right) \perp \operatorname{Mon}\left(\mathcal{H}_{k}\right)$, so the previous Lemma applies. 
We are now ready to prove that every finite abelian group appears as the chirality group of some hypermap. First we deal with cyclic groups:

Proposition 19 For each integer $n \geq 1$ there is an orientably regular hypermap with chirality group $C_{n}$.

Proof We have seen in the proof of Theorem 14 that if $d \geq 3$ and $q$ is sufficiently large, then there are totally chiral hypermaps $\mathcal{K}$ and $\mathcal{H}$ with normal hypermap subgroups $K \leq H$ in $\Delta^{+}$satisfying $\Delta^{+} / K \cong X=S L_{d}(q), \Delta^{+} / H \cong \bar{X}=P S L_{d}(q)$, and $H / K \cong Z=Z(X)$. By Proposition $16, Z \cong X(\mathcal{L})$ where $\mathcal{L}=\mathcal{K} \times \mathcal{H}^{r}$. Now $Z$ is a cyclic group of order $\operatorname{gcd}(q-1, d)$, and we can choose $d(\geq 3)$ and $q$ (sufficiently large) so that this takes any prescribed value $n \geq 1$ : if $n \geq 3$ we can take $d=n$ and $q \equiv 1(\bmod n)\left(\right.$ recall that Euler's Theorem gives $p^{\phi(n)} \equiv 1(\bmod n)$ for each prime $p$ not dividing $n)$, and if $n=1$ or 2 we can take $d=4$ and $q \equiv 0$ or $3(\bmod 4)$, respectively.

We now extend this method of proof to all abelian groups:

Theorem 20 Every finite abelian group is the chirality group of some orientably regular hypermap.

Proof Every finite abelian group can be written in the form $A=C_{n_{1}} \times \cdots \times C_{n_{k}}$ for some positive integers $n_{1}, \ldots, n_{k}$. By the proof of Theorem 14, we can choose normal subgroups $K_{i} \leq H_{i}$ of $\Delta^{+}$for $i=1, \ldots, k$ such that $\Delta^{+} / K_{i} \cong S L_{d_{i}}\left(q_{i}\right), \Delta^{+} / H_{i} \cong$ $P S L_{d_{i}}\left(q_{i}\right)$ and $H_{i} / K_{i}=Z\left(\Delta^{+} / K_{i}\right) \cong C_{n_{i}}$ with $d_{i} \geq 3$, and the corresponding orientably regular hypermaps $\mathcal{K}_{i}$ and $\mathcal{H}_{i}$ are totally chiral; moreover, if $n_{i}=n_{j}$ for some $i \neq j$ then we can take $q_{i} \neq q_{j}$. Now every proper normal subgroup of $S L_{d_{i}}\left(q_{i}\right)$ is central, so every non-trivial epimorphic image of $S L_{d_{i}}\left(q_{i}\right)$ maps onto $P S L_{d_{i}}\left(q_{i}\right)$; for distinct pairs $d_{i}(\geq 3)$ and $q_{i}$ the simple groups $P S L_{d_{i}}\left(q_{i}\right)$ are non-isomorphic, so they are mutually orthogonal, and hence so are the groups $S L_{d_{i}}\left(q_{i}\right)$.

By Corollary 18, the hypermaps $\mathcal{H}=\mathcal{H}_{1} \vee \cdots \vee \mathcal{H}_{k}=\mathcal{H}_{1} \times \cdots \times \mathcal{H}_{k}$ and $\mathcal{K}=\mathcal{K}_{1} \vee \cdots \vee \mathcal{K}_{k}=\mathcal{K}_{1} \times \cdots \times \mathcal{K}_{k}$ are both totally chiral, corresponding to normal subgroups $H=H_{1} \cap \cdots \cap H_{k}$ and $K=K_{1} \cap \cdots \cap K_{k}$ of $\Delta^{+}$with $H / K \cong$ $\left(H_{1} / K_{1}\right) \times \cdots \times\left(H_{k} / K_{k}\right) \cong A$. By Proposition 16 the hypermap $\mathcal{L}=\mathcal{K} \times \mathcal{H}^{r}$ corresponding to $L=K \cap H^{r}$ has $X(\mathcal{L}) \cong H / K \cong A$.

\section{Nonabelian chirality groups}

The preceding results make it tempting to conjecture that every finite group is the chirality group of some orientably regular hypermap; for instance, Lemma 11 shows that every simple group with an asymmetric generating pair arises in this way. However, the next result shows that such a conjecture is false. For any group $S$, let $Z, I$ and $A$ denote the centre $Z(S)$, the inner automorphism group $\operatorname{Inn} S \cong S / Z$, and the automorphism group Aut $S$. The action of $S$ by conjugation on itself induces a homomorphism $S \rightarrow I \leq A$ with kernel $Z$; for each subgroup $T \leq S$ let $\bar{T}$ denote the 
image $T Z / Z$ of $T$ in $I$. A subgroup $T \leq S$ is characteristic in $S$ if it is invariant under $A$; this implies that $\bar{T}$ is a normal subgroup of $A$.

Theorem 21 If a group $S$ has a characteristic subgroup $T$ such that $\bar{T}<\bar{S}$ and $A / \bar{T}$ is abelian, then $S$ cannot be a chirality group.

Before proving this result, let us examine some of its consequences. Recall that a group $S$ is complete if $S$ has trivial centre and all its automorphisms are inner, so that $S \cong$ Aut $S$.

Corollary 22 If $S$ is a complete group and $S$ is not perfect, then $S$ cannot be a chirality group.

Proof Let $T$ be the commutator subgroup $S^{\prime}$, so $T<S$ since $S$ is not perfect. Since $Z=1$ we have $\bar{S}=S$ and $\bar{T}=T$, so $\bar{T}<\bar{S}$. Since $A=S$ we have $A / \bar{T}=S / S^{\prime}$, which is abelian, so Theorem 21 gives the result.

Corollary 23 The symmetric group $S_{n}$ is a chirality group if and only if $n \leq 2$.

Proof Let $S=S_{n}$ for any $n>2$, and take $T$ to be the alternating group $A_{n}$, a proper subgroup of $S$ which is characteristic since $T=S^{\prime}$. We have $Z=1$, and if $n \neq 6$ then $A=S$, so Corollary 22 implies that $S$ cannot be a chirality group. If $n=6$ then $|A: S|=2$, so $A / \bar{T}=A / T$ is abelian (of order 4) and Theorem 21 gives the required result. However, $S_{n} \cong C_{n}$ for $n=1$ and 2 , and these are chirality groups by Proposition 19.

More generally, the automorphism group $S$ of a nonabelian finite simple group $F$ is always complete, by a theorem of Burnside [28, Theorem 13.5.9]. In many cases $F$ has outer automorphisms, so $S>F$; by the Schreier Conjecture, the proof of which follows from the classification of finite simple groups [16, Theorem 1.46], $S / F$ is solvable, so $S$ is not perfect and hence cannot be a chirality group.

Corollary 24 The dihedral group $D_{n}$ is a chirality group if and only if $n \leq 2$.

Proof Let $S$ be the dihedral group $D_{n}=\left\langle a, b \mid a^{n}=b^{2}=(a b)^{2}=1\right\rangle$, with $n>2$, and take $T=\langle a\rangle \cong C_{n}$. Since $T$ is generated by the elements of order $n$ in $S$, it is a characteristic subgroup of $S$. Now $A=$ Aut $S$ is isomorphic to the holomorph of $C_{n}$, a split extension of $C_{n}$ by Aut $C_{n}=U_{n}$, the group of units $\bmod (n)$ : the normal subgroup $C_{n}$ is generated by the automorphism fixing $a$ and sending $b$ to $a b$, while the complement $U_{n}$ is the automorphism group of $T$, fixing $b$. If $n$ is odd, then $Z=1$, so $\bar{S}=S$ and $\bar{T}=T$; since $A / \bar{T}$ is isomorphic to $U_{n}$ it is abelian, so Theorem 21 implies that $S$ cannot be a chirality group. If $n=2 m$ is even, then $Z=\left\langle a^{m}\right\rangle \cong C_{2}$, so $\bar{S} \cong D_{m}$ and $\bar{T} \cong C_{m}$; in this case, $A / \bar{T} \cong U_{n} \times C_{2}$ which is again abelian, so we have the same result.

The above proof fails for $n=2$ since $C_{2}$ is not characteristic in $D_{2}$; indeed, $D_{n}$ is abelian for $n \leq 2$, and is therefore a chirality group by Theorem 20 . 
Corollary 25 If $q=p^{e}$ where $p$ is prime, and divides $p-1$, then $P G L_{d}(q)$ and $G L_{d}(q)$ are not chirality groups.

Proof Take $S=P G L_{d}(q)$ and $T=P S L_{d}(q)$, so $Z=1$ and $\bar{S} / \bar{T}=S / T \cong$ $F_{q}^{*} /\left(F_{q}^{*}\right)^{d} \cong C_{(d, q-1)}$. We have $A=P \Gamma L_{d}(q)$ with $A / T$ abelian (isomorphic to $\left.C_{(d, q-1)} \times C_{e}=C_{d} \times C_{e}\right)$ if $d$ divides $p-1$, in which case $P G L_{d}(q)$ cannot be a chirality group by Theorem 21 . The same applies to $S=G L_{d}(q)$, with $T=S L_{d}(q)$ and $Z$ the group of scalar matrices, so that $\bar{S}=P G L_{d}(q)$ and $\bar{T}=P S L_{d}(q)$.

In particular, if $q$ is odd then $P G L_{2}(q)$ and $G L_{2}(q)$ are not chirality groups.

Proof of Theorem 21 Suppose that $S$ is a chirality group, so $\Delta^{+}$has a normal subgroup $H$ with $H / H_{\Delta} \cong H^{r} / H_{\Delta} \cong H^{\Delta} / H \cong H^{\Delta} / H^{r} \cong S$, in the usual notation. Let $Q=\Delta^{+} / H_{\Delta}$, so $Q$ has normal subgroups $N_{1}=H / H_{\Delta}$ and $N_{2}=H^{r} / H_{\Delta}$, both isomorphic to $S$, generating their direct product $N_{1} \times N_{2} \cong S \times S$ as a normal subgroup $N=H^{\Delta} / H_{\Delta}$ of $Q$. Now let $C$ be the centraliser $C_{Q}(N)$, a normal subgroup of $Q$, and for each subgroup $X \leq Q$ let $\bar{X}=X C / C \cong X /(X \cap C)$, the image of $X$ in $Q / C$. We have $N \cap C=Z\left(N_{1}\right) \times Z\left(N_{2}\right) \cong Z(S)^{2}$, so $\bar{N}=\bar{N}_{1} \times \bar{N}_{2} \cong(S / Z(S))^{2}$, with $\bar{N}$ and each $\bar{N}_{i}$ normal in $\bar{Q}$. The action of $Q$ by conjugation on $N$, preserving each $N_{i}$, induces a faithful action of $\bar{Q}$; this gives an embedding $\bar{Q} \leq$ Aut $N_{1} \times$ Aut $N_{2} \cong A^{2}$, where $A$ denotes Aut $S$.

In the natural homomorphism $S \rightarrow \bar{S}=S / Z(S)=\operatorname{Inn} S \leq A$, where $S$ acts on itself by conjugation, any characteristic subgroup $T$ of $S$ maps to a normal subgroup $\bar{T}$ of $A$, contained in $\bar{S}$. Suppose that $\bar{T}<\bar{S}$ and $A / \bar{T}$ is abelian. If $\bar{T}_{i}$ is the subgroup of $\bar{N}_{i}$ corresponding to $\bar{T}$, then $\bar{T}_{1} \times \bar{N}_{2}$ is a normal subgroup of $\bar{Q}$, with

$$
\bar{Q} /\left(\bar{T}_{1} \times \bar{N}_{2}\right) \leq A^{2} /\left(\bar{T}_{1} \times \bar{N}_{2}\right) \cong A / \bar{T}_{1} \times A / \bar{N}_{2} .
$$

Now $A / \bar{T}_{1}$ is abelian, as is its quotient $A / \bar{N}_{2}$, and hence so is $\bar{Q} /\left(\bar{T}_{1} \times \bar{N}_{2}\right)$. The automorphism $\alpha$ of $\Delta^{+}$acts on $Q$, preserving $N$ and hence $C$, so it induces an automorphism of $\bar{Q}$, inverting its two generators; since $\bar{Q} /\left(\bar{T}_{1} \times \bar{N}_{2}\right)$ is abelian, it has an automorphism inverting its two generators, so $\alpha$ preserves $\bar{T}_{1} \times \bar{N}_{2}$. However, it transposes $\bar{N}_{1}$ and $\bar{N}_{2}$ and hence transposes $\bar{T}_{1} \times \bar{N}_{2}$ and $\bar{N}_{1} \times \bar{T}_{2}$, which are distinct since $\bar{T}_{i}<\bar{N}_{i}$. This contradiction shows that $S$ cannot be a chirality group.

A similar argument shows that $A_{5}$ cannot be a chirality group. For otherwise, putting $S=A_{5}$ we have $Z(S)=1$ and $A=S_{5}$, so $A_{5} \times A_{5} \leq \bar{Q} \leq S_{5} \times S_{5}$ with $\alpha$ inducing an automorphism of $\bar{Q}$ which transposes the two copies of $A_{5}$. This implies that $\Delta^{+}$has a normal subgroup, with quotient group $A_{5}$ or $S_{5}$, which is not normal in $\Delta$, contradicting Corollary 9.

In contrast with the rather simple criteria in Theorem 20 and Corollaries 23 and 24 the next result suggests that a complete characterisation of chirality groups will be a difficult task.

Theorem 26 The 1-dimensional affine group $A G L_{1}(q)$ is a chirality group if and only if $q$ is an odd power of 2. 
Proof If $S=A G L_{1}(q)$ then $Z=Z(S)=1$, and we can identify $A=$ Aut $S$ with $A \Gamma L_{1}(q)$. This group consists of the transformations $t \mapsto a t^{\gamma}+b$ of $F_{q}$, where $a, b \in F_{q}, a \neq 0$ and $\gamma \in \Gamma=\mathrm{Gal} F_{q}$; this Galois group is a cyclic group of order $e$ generated by the Frobenius automorphism $t \mapsto t^{p}$ of $F_{q}$, where $q=p^{e}$ and $p$ is prime. The transformations with $\gamma=1$ form the group $S$, a normal subgroup of index $e$ in $A$ on which $A$ acts by conjugation.

In the proof we will use the following subgroups of $S$ : for each divisor $d$ of $q-1$ let $T_{d}$ be the group of transformations $t \mapsto a t+b$ where $a$ is a $d$-th power in $F_{q}^{*}$. This is the unique subgroup of index $d$ in $S$, so it is characteristic in $S ; A / T_{d}$ is a split extension of $S / T_{d} \cong C_{d}$ by $A / S \cong \Gamma \cong C_{e}$.

If $q$ is odd, we can apply Theorem 21 with $T=T_{2}$. We have $\bar{S}=S$ and $\bar{T}=T$, so $\bar{T}<\bar{S}$. Since $A / \bar{T}=A / T \cong C_{2} \times C_{e}$, which is abelian, Theorem 21 implies that $S$ cannot be a chirality group.

Now let $q=2^{e}$. First we show that if $e$ is odd then $S$ is a chirality group. Since $A G L_{1}(2) \cong C_{2}$, we may assume that $e>1$. Let $x \in A$ be given by $t \mapsto t^{2}$, and let $y \in S$ be given by $t \mapsto u(t-v)+v=u t+(1-u) v$, where $u$ generates $F_{q}^{*}$ and $v \in F_{q} \backslash F_{2}$. Then $y$ generates the stabiliser $S_{v}$ in $S$ of $v$, a cyclic group of order $q-1$ consisting of the transformations $t \mapsto u^{i}(t-v)+v$, and its conjugate $y^{x}=x^{-1} y x: t \mapsto(u \sqrt{t}+(1-u) v)^{2}=u^{2} t+(1-u)^{2} v^{2}$ generates the stabiliser $S_{v^{2}}$ of $v^{2}$. Since $v \neq 0,1$ we have $v \neq v^{2}$, so $S_{v} \neq S_{v^{2}}$. Since $S$ acts primitively (in fact, doubly transitively) on $F_{q}$, the stabilisers of points are maximal subgroups of $S$, so $S=\left\langle S_{v}, S_{v^{2}}\right\rangle=\left\langle y, y^{x}\right\rangle$; since $x S$ generates $A / S$ it follows that $A=\left\langle x, y, y^{x}\right\rangle=$ $\langle x, y\rangle$. This gives an epimorphism $\theta: \Delta^{+} \rightarrow A$, so that $A$ is the monodromy group of a regular orientable hypermap, corresponding to the normal subgroup $H=\operatorname{ker} \theta$ of $\Delta^{+}$.

The normal subgroup $K=\theta^{-1}(S)$ of $\Delta^{+}$satisfies $\Delta^{+} / K \cong A / S \cong \mathrm{Gal} F_{q} \cong C_{e}$, so $\Delta^{+} / K$ is abelian; it follows from Corollary 9 that $K$ is normal in $\Delta$ and hence $H^{\Delta} \leq K$. If we can show that $H^{\Delta}=K$ then we have a chirality group $H^{\Delta} / H=K / H \cong S$, as required, so suppose that $H^{\Delta}<K$. Since $H^{\Delta} \geq H$ we have $H^{\Delta}=\theta^{-1}(N)$ for some normal subgroup $N$ of $A$, properly contained in $S$, and hence contained in a maximal normal subgroup of $S$. Now the maximal normal subgroups of $S$ are the subgroups $T_{p}$ defined earlier, one for each prime $p$ dividing $q-1$, so $N \leq T_{p}$ for some $p$. Since $q$ is even, $p$ is odd. We have

$$
A / T_{p}=\left\langle x, y \mid x^{e}=y^{p}=1, y^{x}=y^{2}\right\rangle,
$$

so in $A / T_{p}$ we have

$$
\left(y^{-1}\right)^{x^{-1}}=y^{-1 / 2}\left(=y^{(p-1) / 2}\right) .
$$

Now the automorphism $\alpha$ of $\Delta^{+}$leaves $H^{\Delta}$ and $K$ invariant, so it induces an automorphism of $A / N\left(\cong \Delta^{+} / H^{\Delta}\right)$ leaving $S / N\left(\cong K / H^{\Delta}\right)$ invariant; since $T_{p} / N$ is the unique normal subgroup of index $p$ in $S / N$ it is invariant under $\alpha$, which therefore induces an automorphism of $A / T_{p}$. This automorphism inverts $x$ and $y$, so by applying it to the relation $y^{x}=y^{2}$ of $A / T_{p}$ we get

$$
\left(y^{-1}\right)^{x^{-1}}=y^{-2}
$$


in $A / T_{p}$. Comparing these two equations we get $y^{-1 / 2}=y^{-2}$, so $y^{3}=1$ in $A / T_{p}$ and hence $p=3$. Thus $q=2^{e} \equiv 1 \bmod (3)$, so $e$ is even, against our hypothesis. This contradiction shows that $A G L_{1}\left(2^{e}\right)$ is a chirality group for all odd $e$.

If $e=2 f$ is even then $q \equiv 1 \bmod (3)$, so $S$ has a characteristic subgroup $T=T_{3}$ with

$$
A / T=\left\langle x, y \mid x^{e}=y^{3}=1, y^{x}=y^{2}\right\rangle
$$

and $S / T=\langle y\rangle \cong C_{3}$. The subgroup $\left\langle x^{2}\right\rangle$, isomorphic to $C_{f}$, is normal (in fact, central) in $A / T$, with quotient isomorphic to $D_{3}$; let $U$ denote its inverse image in $A$, so $T \leq U \leq A$ with $U / T \cong C_{f}$ and $A / U \cong D_{3}$. For any subgroup $X$ of $A$ (or of $A^{2}$ ), let $X^{*}$ denote its image $X U / U \cong X /(X \cap U)$ in $A / U$ (or in $A^{2} / U^{2}$ ), so $S^{*} \cong C_{3}$ and $A^{*} \cong D_{3}$. We have $T^{2}<S^{2} \leq \bar{Q} \leq A^{2}$, so $C_{3}^{2} \cong\left(S^{2}\right)^{*} \leq \bar{Q}^{*} \leq\left(A^{2}\right)^{*} \cong D_{3}^{2}$ and hence $\bar{Q}^{*}$ is either $C_{3}^{2}$ or $D_{3}^{2}$ or an extension of $C_{3}^{2}$ by $C_{2}$. Now $\alpha$ transposes $H$ and $H^{r}$, so it induces an automorphism of $\bar{Q}^{*}$, inverting its generators and transposing the corresponding two normal subgroups $C_{3}$. However, these two normal subgroups of $\bar{Q}^{*}$ have quotient group $C_{3}$ or $D_{3}$ or $D_{3} \times C_{2} \cong D_{6}$, which are abelian or dihedral, so in each case Proposition 8 implies that they are invariant under $\alpha$. This contradiction shows that $A G L_{1}\left(2^{e}\right)$ is not a chirality group when $e$ is even.

Acknowledgement The authors are grateful to the referees for their careful reading of the paper and their very helpful suggestions and corrections.

\section{References}

1. Barron, L.D.: Fundamental symmetry aspects of molecular chirality. In: Mezey, P.G. (ed.) New Developments in Molecular Chirality. Kluwer Academic, Dordrecht (1991)

2. Breda, A., Breda d'Azevedo, A., Nedela, R.: Chirality index of Coxeter chiral maps. Ars Comb. 81, 147-160 (2006)

3. Breda d'Azevedo, A.J., Jones, G.A.: Double coverings and reflexible abelian hypermaps. Beiträge Algebra Geom. 41, 371-389 (2000)

4. Breda d'Azevedo, A., Nedela, R.: Chiral hypermaps with few hyperfaces. Math. Slovaca 53(2), 107128 (2003)

5. Brown, C. (ed.): Chirality in Drug Design and Synthesis. Academic Press, London (1990)

6. http://www.math.auckland.ac.nz/ conder/

7. Conder, M.D.E., Dobcsányi, P.: Determination of all regular maps of small genus. J. Combin. Theory Ser. B 81, 224-242 (2001)

8. Conway, J.H., Curtis, R.T., Norton, S.P., Parker, R.A., Wilson, R.A.: ATLAS of Finite Groups. Clarendon, Oxford (1985)

9. Cori, R., Machi, A.: Maps, hypermaps and their automorphisms: a survey I. Expo. Math. 10, 403-427 (1992)

10. Cori, R., Machi, A.: Maps, hypermaps and their automorphisms: a survey II. Expo. Math. 10, 429-447 (1992)

11. Cori, R., Machi, A.: Maps, hypermaps and their automorphisms: a survey III. Expo. Math. 10, 449467 (1992)

12. Coxeter, H.S.M., Moser, W.O.J.: Generators and Relations for Discrete Groups, 3rd edn. Springer, New York (1972)

13. Corn, D., Singerman, D.: Regular hypermaps. Eur. J. Comb. 9, 337-351 (1988)

14. Dieudonné, J.: La Géométrie des Groupes Classiques. Springer, Berlin (1955)

15. Fujita, S.: Symmetry and Combinatorial Enumeration in Chemistry. Springer, Berlin (1991)

16. Gorenstein, D.: Finite Simple Groups. Plenum, New York (1982) 
17. Guralnick, R., Kantor, W., Saxl, J.: Probability of generating a classical group. Commun. Algebra 22, 1395-1402 (1994)

18. Huppert, B., Blackburn, N.: Finite Groups III. Springer, Berlin (1982)

19. Janoschek, R. (ed.): Chirality-From Weak Bosons to $\alpha$-Helix. Springer, Berlin (1991)

20. Jones, G.A.: Ree groups and Riemann surfaces. J. Algebra 165, 41-62 (1994)

21. Jones, G.A., Silver, S.A.: Suzuki groups and surfaces. J. Lond. Math. Soc. 48(2), 117-125 (1993)

22. Lord Kelvin: Baltimore Lectures. C.J. Clay, London (1904)

23. Macbeath, A.M.: Generators of the linear fractional groups. In: Number Theory (Proc. Sympos. Pure Math., Vol. XII, Houston, Tex., 1967), pp. 14-32. Amer. Math. Soc., Providence (1967)

24. Mezey, P.G. (ed.): New Developments in Molecular Chirality. Kluwer Academic, Dordrecht (1991)

25. Ree, R.: A family of simple groups associated with the simple Lie algebra of type $\left(G_{2}\right)$. Bull. Am. Math. Soc. 66, 508-510 (1960)

26. Ree, R.: A family of simple groups associated with the simple Lie algebra of type $\left(G_{2}\right)$. Am. J. Math. 83, 432-462 (1961)

27. Singerman, D.: Symmetries of Riemann surfaces with large automorphism group. Math. Ann. 210, 17-32 (1974)

28. Robinson, D.J.S.: A Course in the Theory of Groups. Springer, New York (1982)

29. Suzuki, M.: On a class of doubly transitive groups. Ann. Math. 75(2), 105-145 (1962)

30. Walsh, T.R.S.: Hypermaps versus bipartite maps. J. Combin. Theory Ser. B 18, 155-163 (1975)

31. Wielandt, H.: Finite Permutation Groups. Academic Press, New York (1964) 